\title{
The Symplasmic Organization of the Shoot Apical Meristem
}

C. VAN DER SCHOOT ${ }^{1} \cdot$ P. RINNE ${ }^{2, *}$

${ }^{1}$ ATO-DLO, P.O. Box 17, NL-6700 AA Wageningen, The Netherlands

2 Department of Virology, Agricultural University Wageningen, Binnenhaven 6, NL-6709 PD Wageningen, The Netherlands

* Permanent address: Department of Biology, University of Oulu, P.O. Box 3000, FIN-90401 Oulu, Finland

1 Introduction 226

2 Shoot Apical Meristem: Structural Aspects 226

2.1 Evolutionary Designs 226

2.2 Monoplex and Duplex 227

2.3 Symplasmic Unity 228

2.4 Tunica 229

2.5 Plasmodesmata: Loci and Modifications 229

3 Shoot Apical Meristem: Supracellular Dynamics 233

3.1 Physiological Barriers 233

3.2 Gene Expression 233

3.3 Macromolecular Trafficking 234

3.4 A Morphogenetic Field 236

3.5 Symplasmic Fields 236

3.6 Symplasmic Field Transitions 238

4 Concluding Remarks 239

References 239 
The buildup of symplasmic organization has been a driving force in the evolution of multicellular plants. A symplasmic organization could arise when walled unicellular ancestors commenced subdividing their protoplasm by means of pore-bearing walls. By restricting the functional size of the pores, cells could develop physiological individuality without giving up cytoplasmic continuity. Further refinement of the pores, leading to the formation of true plasmodesmata, subsequently set the stage for the development of increasingly complex symplasmic organizations. Particularly the appearance of regulatory devices inside the pores, permitting a selective exchange of morphogenetic signals, might have been crucial in the evolution of higher plants (Carr 1976; Gunning and Robards 1976; Robards 1976; Lucas et al. 1993; Franceschi et al. 1994). Since all higher plants arise from the activity of morphogenetic units, the root and shoot meristems, the symplasmic organization of these meristems might play an important role in primary morphogenesis. The symplasmic organization of the shoot apical meristem (AM) channels the flow of signals dependent on the patterns in which cells divide and establish de novo contacts with neighbours. In the following, we will examine how symplasmic networking in the AM might have played a role in the emergence of higher plants, and how it functions in the coordination of morphogenesis.

\section{The Shoot Apical Meristem: Structural Aspects}

\subsection{Evolutionary Designs}

For proper development of the shoot, the AM must not only produce the cells required for growth and development, but also position them in such a way that the basic organization of the AM is maintained. The AM reconciles these tasks by confining the leaf primordia to its periphery, thereby keeping its centre unoccupied. Although this is most clearly so in higher plants, Newman (1965) pointed out that all multicellular plants of the major plant taxa have their growth centres somehow subdivided into a source region and a region of elaboration. The source is the ultimate origin of all cells, probably being identical to the initials. The region of elaboration constitutes the part of the meristem involved in the direct production of leaf buttresses.

In principle, the AM cells are spatially fixed with regard to their neighbours by an extracellular matrix (Roberts 1994), which limits their possibilities for setting up free collaborations. Since collaboration is essential for achieving a common morphogenetic goal, the pattern of cell divisions may have substantial influence on AM functioning. The division of the initial cells and their derivatives leads to specific lineage patterns. The number and position of the initial cells, and the way they restrict the plane of their divisions are dissimilar for the major taxa, i.e. ferns/lower vascular plants, gymnosperms, and angiosperms. This observation led Newman (1965) to classify their AMs as monoplex, simplex, and duplex, respectively (Fig. 1; see also discussions in Gifford and Corson 1971). The existence of taxonomic differences is informative since it indicates that the distinct AM types represent ontogenetically as well as phylogenetically robust organizations, and that each type of organization suffices for morphogenesis. The fact, however, that the major plant taxa possess shoots with distinct designs rais- 


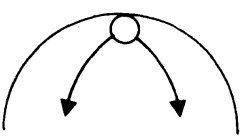

MONOPLEX

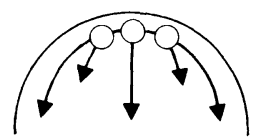

SIMPLEX

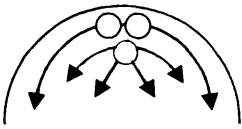

DUPLEX

Fig. 1. Schematic representation of shoot apical meristem (AM) types: monoplex (ferns), simplex (gymnosperms) and duplex (angiosperms). In the monoplex AM, a single, usually tetrahedral, triangular initial cell gives rise to the entire shoot system. In the simplex AM, a superficial layer of initials is present. The duplex AM possesses two sets of initials which give rise to distinct growth zones. (After Newman 1965)

es an important question: to what extent does shoot design result from the type of AM organization? In the following we will therefore examine in greater detail the cell lineage patterns in two distinct AM types.

\subsection{Monoplex and Duplex}

The fern AM has a monoplex construction, i.e. the entire shoot system is derived from a single apical initial cell (Fig. 1). This single cell source is triangular in shape, and the specific way in which it divides by insertion of perpendicular walls to alternating sides of the triangle imposes a characteristic geometrical cell pattern on the emerging tissues (Raghavan 1989; Tilney et al. 1990; Cooke et al. 1996). Since only cytokinetically formed plasmodesmata are present in the monoplex apex, this pattern may be due to the necessity to maintain symplasmic continuity in the shoot system.

In contrast to ferns, the cells in the AM of angiosperms are produced in two structurally or geometrically distinct areas, termed tunica and corpus (Figs. 2A, 3A). This so-called duplex pattern results from the activity of two separate sets of initial cells

Fig. 2 A, B. Schematic representation of models defining morphogenesis at the shoot apical meristem (AM). A Structural organization of the AM reflects the direction of cell divisions (arrows), i.e. anticlinal in the tunica $(t)$ and in all planes in the corpus $(c)$. B Physiological organization is reflected in the cytohistological zonation. Metabolically active cells with high cell cycling $(c c)$ are at the AM periphery $(a)$ and metabolically less active cells with low cell cycling occupy the centre (b). (After Rinne and van der Schoot 1998)
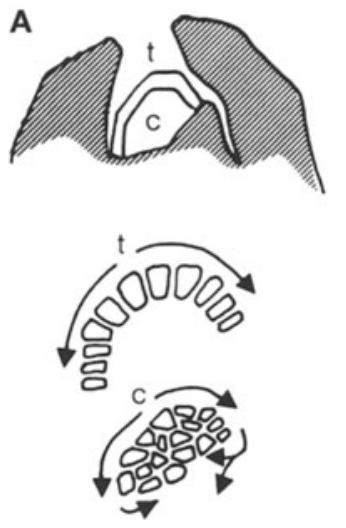
with distinct division patterns (Figs. 1,3A; Gifford and Corson 1971; Tilney-Bassett 1986; Steeves and Sussex 1989). The initials and their derivatives in the tunica show exclusively anticlinal divisions, thereby giving rise to a two-dimensional cell sheet that expands centrifugally. The corpus initials and their derivatives divide in all planes, resulting in the formation of a compact three-dimensional cell group (Tilney-Bassett 1986; Steeves and Sussex 1989; Sachs 1991). The expanding tunica is separated from the corpus by non-division walls which are continuously "stretched and plastered" in the horizontal plane (Roberts 1994). Within the tunica sheet and within the three-dimensional corpus symplasmic continuity of cell lineages is ensured by plasmodesmata formed in the cell plate. The periclinal walls between the tunica and the corpus do not, as a rule, possess cytokinetic plasmodesmata, and thus the numerous plasmodesmata present in this interface must have been made in a different way.

\subsection{Symplasmic Unity}

It may be postulated that the appearance of the duplex AM on the evolutionary stage required the exploitation of a second type of plasmodesmata, one which is formed through existing walls. The occurrence of non-cytokinetic plasmodesmata in angiosperms was proposed as early as 1901 by Strassburger (cited by Carr 1976), who observed that the radial walls of a secondary meristem, the cambium, do not arise as a result of mitosis and yet have plasmodesmata numbers similar to the walls originating from cell plates. Much later, specific cases of such "secondary plasmodesmata" (Jones 1976) in angiosperms were described for developing stem tissues (Seagull 1983; see Chap. 10), graft systems (e.g. Kollmann and Glockmann 1985), and fusing floral organs (van der Schoot et al. 1995). Their presence within the duplex AM, at the interface of tunica and corpus, has remained unnoticed until recently (van der Schoot 1994, 1996; Cooke et al. 1996; van der Schoot and Rinne 1996; Bergmans et al. 1997), although the situation is identical to that of the cambium. Our model shows, in addition, that within the layers and the corpus branches sideways are exclusively interconnected by secondary plasmodesmata (van der Schoot 1996; van der Schoot and Rinne 1996; Bergmans et al. 1997). Resumingly, the plasmodesmata at the tunica/corpus interface, and those between lineages and lineage branches, are all produced through existing walls as a normal and ongoing event during cellular proliferation at the AM. The formation of numerous plasmodesmata at every cell-cell interface of the AM, regardless of cell lineage and cell location (van der Schoot et al. 1991; van der Schoot 1996), is probably a general phenomenon in angiosperms.

The advantage of the subdivision of the AM into the two zones, tunica and corpus, was that their distinct growth dynamics allowed physical stresses to aid phyllotactic patterning by bulging and outgrowth of leaf primordia (e.g. Green 1992; Green et al. 1996). Due to the peripheral positioning of leaf primordia, not involving the central zone of initial cells and their youngest derivatives, the duplex AM gained a potential for indeterminate growth. In addition, secondary plasmodesmata permitted the presence of an entire zone of uncommitted cells (in the tunica as well as in the corpus) instead of a single cell - in which the division frequency could become lower, thereby increasing the longevity of the AM. In contrast, ferns may be limited by the presence of a single initial cell which after a restricted number of divisions becomes senescent, 
resulting in a progressive decrease of plasmodesmata production and, eventually, in cessation of growth (Tilney et al. 1990). Resumingly, the presence of secondary plasmodesmata permitted the introduction of a tunica, which provided the AM of angiosperms with unique properties.

\subsection{Tunica}

The activity of the AM has been characterized as extended embryogenesis (Sachs 1991). From this perspective, the outer wall of the "extended embryo" bears the mark of the zygote since this wall derives directly from it, although stretched and plastered in an endless way (Roberts 1994). All other walls are created as internal walls which "septate" the original zygote (Kaplan and Hagemann 1991; Roberts 1994). One distinctive feature of these outer tunica walls is the complete absence of plasmodesmata (Bergmans et al. 1997). Although plasmodesmata frequently arise in non-division walls and even in single walls, which results in half-plasmodesmata ending blind at the middle lamella (Jones 1976; Ehlers and Kollmann 1996), the outer tunica wall forms no plasmodesmata. (Early discussions on the possible existence of epidermal ecto-plasmodesmata have been reviewed by Carr, 1976.) The lack of plasmodesmata in the outer wall of the tunica, and the presence of exclusively secondary plasmodesmata at the opposite wall, assign a unique polarity to the tunica (Bergmans et al. 1997). It may polarize the organization of the plasmamembrane, membrane-associated cytoskeletal elements, and the cortical ER/plasmodesmata complexes of the entire symplasmic continuum within the AM. This may, for example, facilitate the deposition of materials at the outer walls and the cuticle, and the import and export of macromolecules (see Sect. 3.3).

Since the embryonic wall has been shown to influence cell fate (Berger et al. 1994), it seems feasible that the outer, zygote-derived tunica wall functions somehow as a reference for cellular differentiation (Bergmans et al. 1997). The outer tunica wall may also function as a mechanical framework for the AM due to its relative thickness, whereas the fragile anticlinal walls may serve the spatial partitioning of the protoplasm of the mother cells (Fig. 3A). Although phyllotactic patterning is assisted by biophysical forces in the tunica (e.g. Green 1992; Selker et al.1992; Green et al. 1996), the outgrowth and further development of the leaf primordia requires continuous input of morphogens. Since both, the signalling mechanisms that are utilized in spacing mechanisms such as phyllotactic patterning and those which regulate the dorsiventral development of leaves, act in a radial fashion between the AM centre and the primordia (e.g. Sussex 1952, 1989; Metford et al. 1992), they may resign in the tunica. While serving these purposes, the radially extending tunica layer could have conserved ancient signalling mechanisms, which traffic exclusively through primary, i.e. cytokinetic plasmodesmata.

\subsection{Plasmodesmata: Loci and Modifications}

In the duplex AM symplasmic unity was guaranteed by progressive ways to unite all cells symplasmically. However, a functional subdivision could be achieved if the two 

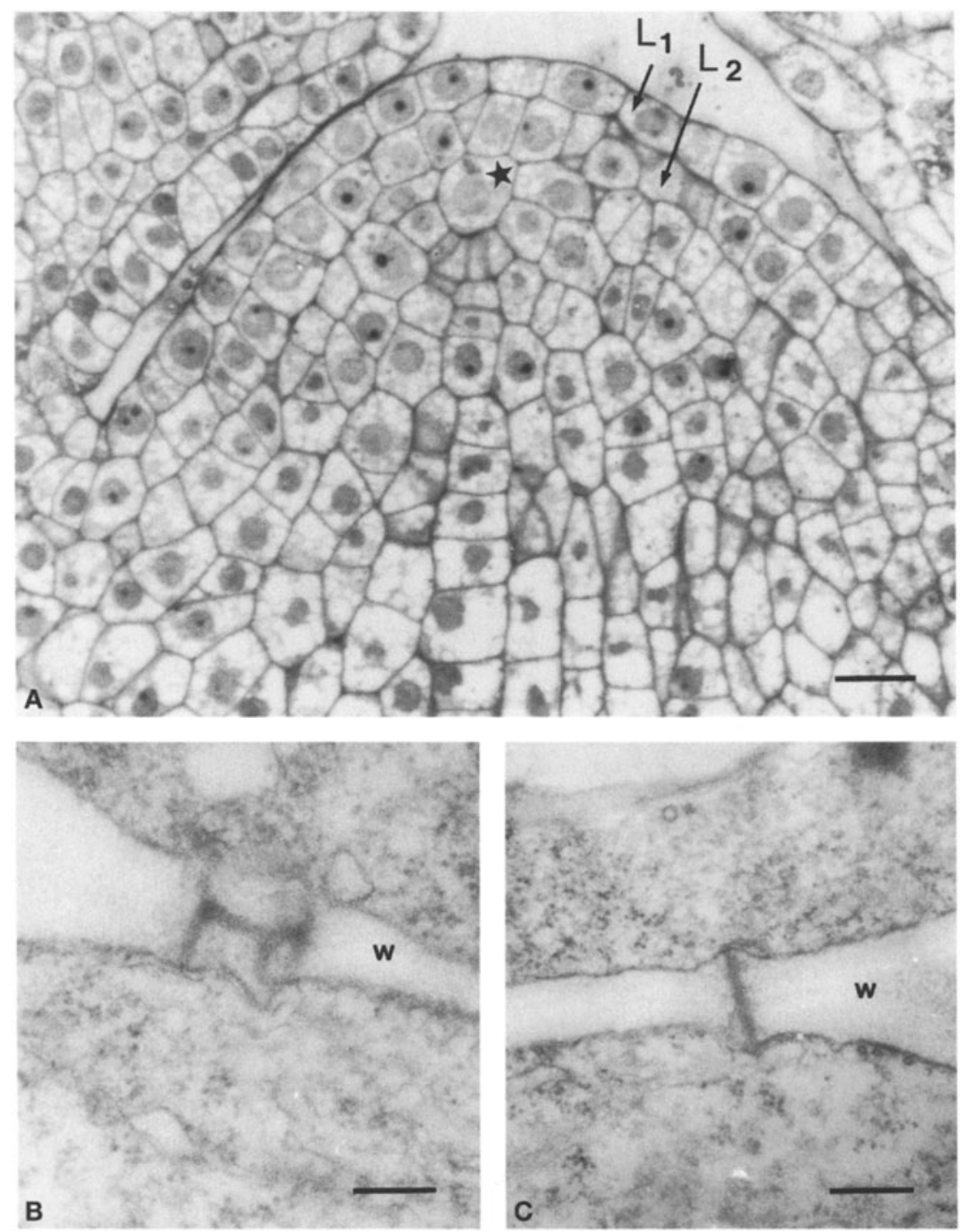

Fig. 3A-C. The inflorescence meristem (IM) of the monocot Iris hollandica and plasmodesmata therein. A A median longitudinal section through the IM shows a two-layered tunica superimposed on a corpus. Note the radiation of cell files from a corpus initial $\left({ }^{*}\right) ; L_{1}$ outer tunica layer; $L_{2}$ second tunica layer. B Branched plasmodesmata, formed by the interconnection of three existing linear secondary plasmodesmata in a periclinal wall $(w)$, between $\mathrm{L}_{1}$ and $\mathrm{L}_{2}$. C Linear plasmodesmata (secondary or primary) in an anticlinal wall $(w)$ of the $\mathrm{L}_{1}$. Bar equals $25 \mu \mathrm{m}(\mathbf{A})$ and $0.25 \mu \mathrm{m}$ (B, C). (Bergmans et al. 1997)

types of plasmodesmata differed in their ability to transfer morphogenetic substances or metabolites from cell-to-cell. For example, signals that are generated in the tuni$\mathrm{ca}$ and traffic through primary plasmodesmata only would be confined to the tunica 
as their trafficking to the subtending corpus would be prevented by the lack of primary plasmodesmata at this interface. However, even when their transfer abilities were identical, a specific distribution of plasmodesmata types within the AM could influence signal flow. This is due to the fact that primary and secondary plasmodesmata are produced by different mechanisms and in different locations, so that their frequencies might be altered independently during development. In the inflorescence meristem of Iris this scenario is not followed, however, and the plasmodesmal frequencies of primary and secondary plasmodesmata decrease simultaneously during the transition to floral meristem (Bergmans et al. 1997). The downregulation of plasmodesmata-forming mechanisms was restricted to the second cell layer within the AM (Fig. 4), showing that the regulation of plasmodesmal frequencies can be locus- rather than type-dependent. Such frequency changes may suffice to alter the flow of morphogenetic signals and thereby morphogenesis, since even the channelling of simple metabolites affects the morphogenetic potential of the various AM areas (Sussex 1952; Steeves and Sussex 1989; Sachs 1991).

It is commonly assumed that branching modifies plasmodesmal function, and therefore, it is important to establish whether it occurs in the AM. Observations indi-
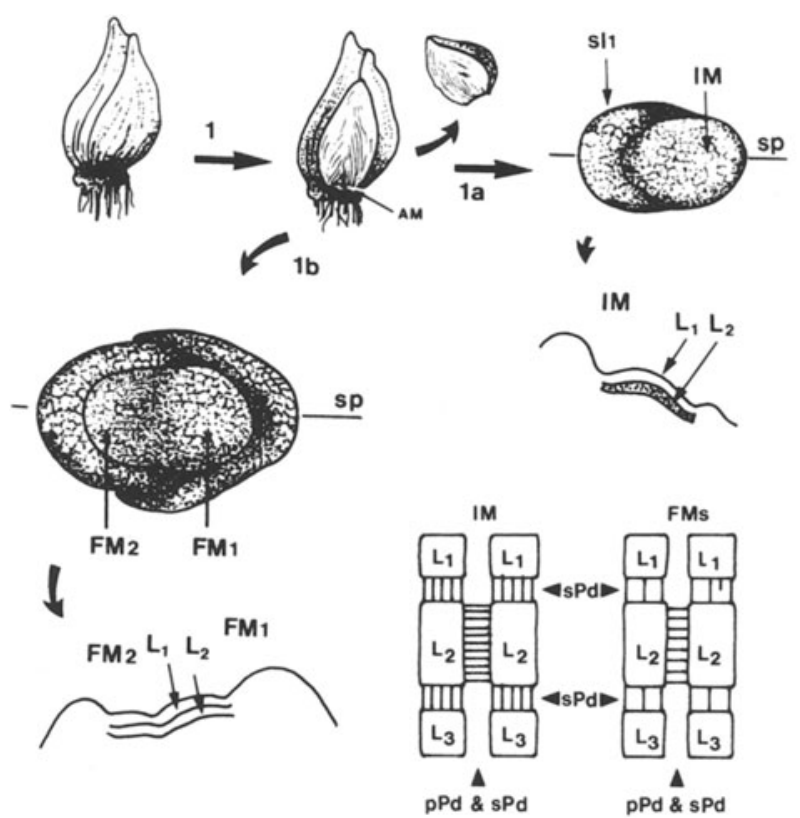

Fig. 4. An Iris bulb cut open longitudinally (1) shows the presence of the shoot apical meristem $(A M)$ at the bottom of the bulb. The AM develops over time ( $1 a)$ into an inflorescence meristem (IM) with a single spathe leaf $\left(s l_{1}\right)$, and further $(1 b)$ into a double floral meristem (FM1 and $F M 2$ ). Schema for longitudinal sections are taken at sp (section plane). Plasmodesmata in the AM showed strong frequency shifts at specific boundaries. A modified plasmodesmogram (bottom right) visualizes the frequency changes occurring during the transition from IM to FM. $p P d$ Primary plasmodesmata; $s P d$ secondary plasmodesmata. Within the first tunica layer $\left(L_{1}\right)$ and within the corpus $\left(L_{3}\right)$, frequencies remained the same. In contrast, plasmodesmal frequencies among the second tunica layer $\left(L_{2}\right)$, between $\mathrm{L}_{2}$ and $\mathrm{L}_{3}$, and between $\mathrm{L}_{2}$ and $\mathrm{L}_{1}$ decreased with 25, 40 and 25\%, respectively. (Bergmans et al. 1997) 
cate that plasmodesmata in the AM can be branched, although the majority of them is linear. In the inflorescence meristem of Iris, for example, virtually all plasmodesmata are linear (Fig. 3B, C; Bergmans et al. 1997). Similarly, in the floral meristem of Helianthus annuus L. only few branched plasmodesmata are present (Sawhney et al. 1981). In potato (Solanum tuberosum L.), however, the plasmodesmata between tunica and corpus tend to branch, whereas the plasmodesmata formed within layers are almost exclusively linear (C. van der Schoot, unpub. results). The lack of branched plasmodesmata in most meristems indicates that branched plasmodesmata are not required for AM functioning. The occasional branching of plasmodesmata in the AM may simply reflect the response of the aligning cells to unequal wall stretching (Fig. 5) and the necessity to maintain the symplasmic unity of the meristem. The tendency to branching between tunica and corpus and the lack of it within the tunica would then indicate that differential wall stretching is stronger between tunica and corpus than within the tunica.

It has been speculated that branched plasmodesmata have a special ability to traffic so-called informational molecules, while linear plasmodesmata do not have this ability (Ding et al. 1992, 1993). There seems to be no reason, however, why the macromolecular complex intrinsic to branched plasmodesmata could not be present in linear plasmodesmata (primary or secondary). The plasmodesmata which transport the necessary signals for the fusion of flower carpels also do not seem to require exclusive-

A

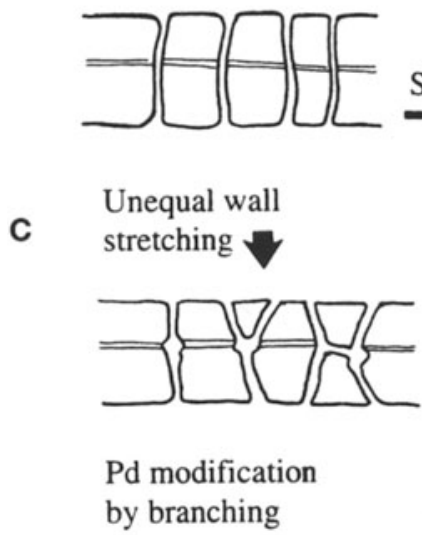

B

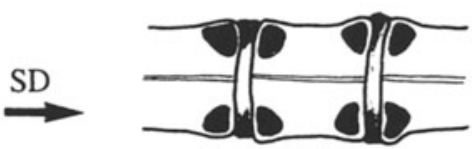

Sphincters

D

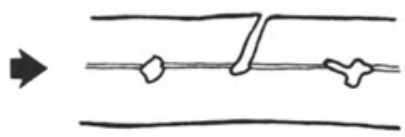

Secondary parts of modified pd

Fig. 5A-D. Schematic representation of plasmodesmata modifications in the angiosperm AM. A Cellcell interfaces cemented together by a middle lamella. Plasmodesmata are linear and may represent either primary plasmodesmata formed in a cell plate or secondary plasmodesmata formed at the interface of non-doughter cells. B Short photoperiod $(S D)$ triggers mechanisms that result in modification of plasmodesmata as observed in the birch AM (Rinne and van der Schoot 1998). The structural modifications resemble sphincters (cf. Olesen and Robards 1990) and serve the temporary symplasmic isolation of all AM cells. C Unequal stretching of periclinal tunica walls leads to morphological modifications of existing plasmodesmata. Plasmodesmata may form small median cavities, new branches, or interconnect two or three existing plasmodesmata. D Projection (from C) of the newly constructed parts of modified plasmodesmata. In case such additions have different functional properties, this in most cases will not lead to alterations in cell-to-cell transfer, since the new parts do not cross both cell walls entirely. Pd, plasmodesmata 
ly linear or branched plasmodesmata, since both morphotypes of secondary plasmodesmata are present (van der Schoot et al. 1995).

Another modification of the plasmodesmata which may occur in the AM is the formation of valve-like structures at the plasmodesmata entrances (Fig. 5; van der Schoot 1994, 1996; Rinne and van der Schoot 1998). Earlier, these structures, found in a variety of other plant parts, were depicted as flux-regulating sphincters (Olesen and Robards 1990) or as defence structures formed during a stress response (Turner et al. 1994). It has been shown with microinjection techniques that in the AM of birch they function as valves which physically close the plasmodesmata, resulting in the symplasmic isolation of all AM cells (Rinne and van der Schoot 1998). The production of valves on the plasmodesmata appears to be an integral part of the dormancy mechanism (discussed in Sect. 3.6). It remains to be seen if their occurrence is a more universal response against environmental stress.

\section{Shoot Apical Meristem: Supracellular Dynamics}

\subsection{Physiological Barriers}

Newman's classification of AM types (1965) is based on differences in the cellular construction patterns (Fig. 1). Equally important is a model describing the AM in terms of physiological patterns, known as the cytohistological zonation-model (Fig. 2B; Steeves and Sussex 1989). In most seed plants, cytohistological zones are present in the AM, which are discernible by their staining properties, organelle distributions and cell cycling times (Steeves and Sussex 1989; Sachs 1991). The zones are present as a central disc and a peripheral ring, which include several superimposed layers. A further subdivision can be found within the central zone. For example, in sunflower, the tunica layer of the central zone has a cytohistological appearance that is distinct from the underlying corpus layers within the central zone (Sawhney et al. 1981). The distinct cell groups and subgroups suggest the presence of multiple physiological barriers inside the AM (Sawhney et al. 1981; Bergmans et al. 1993). Recent evidence shows that the barriers in the tunica may result from the narrowing of the plasmodesmal channels at specific locations between the peripheral and the central area of the AM and between the tunica and the corpus (see Sect. 3.5; Rinne and van der Schoot 1998).

The physiological barriers match the division of tasks within the AM, and may separate the processes of primordium formation and self-renewal (van der Schoot 1996; van der Schoot and Rinne 1996). It would be of interest to establish whether and how this physiological subdivision relates to gene expression during morphogenesis and morphogenetic switching.

\subsection{Gene Expression}

During vegetative growth, gene expression patterns in the AM are relatively stable, and no major developmental shifts occur. The spatially restricted activation of homeotic genes such as knox3 and $r s 1$ in maize (Jackson et al. 1994) and nam in petunia (Souer et al. 1996) may serve the specification of primordia and their separation from the AM 
centre, respectively. The expression domain of the homeobox gene knotted 1 in maize, important for indeterminate growth, is also restricted and does not include the tunica layer and the future primordia sites as judged from in situ hybridization experiments (Jackson et al. 1994). However, the KNOTTED 1 protein is detected in the tunica, and is proposed to be imported from lower cell layers. Some other spatial differences occur in the expression of "house-hold" genes (Fleming et al. 1992, 1993; Pri-Hadash et al. 1992). Their differential expression in tunica or corpus, central or peripheral zone, reflects differences in metabolism, possibly serving the division of tasks in the AM.

The area-wise expression of various regulatory genes in the vegetative meristem may be altered during the transition to flowering (e.g. Kelly et al. 1990; Pri-Hadash et al. 1992; Menzel et al. 1996). For example, in mustard (Sinapis alba) the expression of the mADS box genes samads A and samads B is restricted to the central zone in the vegetative meristem, but after the transition it spreads out to the peripheral zone (Menzel et al. 1996). This might reflect the disappearance of the cytohistological zonation pattern and a corresponding change in the symplasmic organization of the AM. Concommitantly, the AM alters its gene expression patterns to canalize he further development of a flower by a sequential expression of regulatory genes (e.g. Coen and Meyerowitz 1991; Weigel and Clark 1996).

An important problem, which deserves attention, is how the patterns are maintained since the AM cells are collectively displaced towards the periphery, and their gene expression, cell cycling and cytological characteristics change accordingly. The persistence of these patterns implies that the cells continuously communicate their position to each other and to newcomers. Although little is known about the kind of signals that are exchanged in these interactions, recent speculations assign an important role to macromolecular transfer in development.

\subsection{Macromolecular Trafficking}

The transcription factor KNOTTED 1, a homeodomain protein, is suggested to move selectively through plasmodesmata in the AM of maize and facilitate also the movement of its own mRNA (Jackson et al. 1994; Lucas et al. 1995). Fluorescently tagged KNOTTED 1 , microinjected into mesophyll cells of maize and tobacco, moved to all neighbouring cells, indicating that it moved through primary and secondary plasmodesmata. Movement of KNOTTED 1, therefore, seems independent of plasmodesmata type (primary, secondary), tissue status (meristematic, mature) and plant taxa (the monocotyledon maize and the dicotyledon tobacco), and demonstrates lack of selectivity. It is possible, of course, that the plasmodesmata in the mesophyll are not representative, since their major function is assimilate transfer, and that in meristematic tissues such selectivity plays a role. In the AM, the protein, but not its mRNA, is present in the tunica, whereas both are absent from the tunica derived leaf buttress (Lucas et al. 1995). Since both, tunica and leaf buttress, are connected by secondary plasmodesmata to KNOTTED 1 producing cells, selectivity may be due to physiological and physical factors that determine whether specific functions of plasmodesma are actually executed.

Also in the floral meristem macromolecular trafficking through plasmodesmata could possibly occur. This might be inferred from the observation that certain genes function non-cell-autonomously, and their downstream effects are triggered also in 
neighbouring cells that do not express the gene themselves (Becraft 1995). In some cases, like deficiens (def) and globosa ( $g l o$ ) in Antirrhinum, this interaction is polar in the sense that lower layers affect cells in the tunica but not vice versa (Perbal et al. 1996). In other cases, like that of floricaula (flo) in Arabidopsis, interactions are opposite, i.e. from the tunica towards the corpus (Furner et al. 1996). In case of FLO it is unclear whether the protein itself traffics between the tunica and the corpus (e.g. Jackson et al. 1994), or whether as yet unidentified factors mediate this non-cell-autonomous gene expression (Hake and Freeling 1986; Jackson et al. 1994; Carpenter and Coen 1995; Hantke et al. 1995; Furner et al. 1996; Perbal et al. 1996). FLo or its mediating factors may be transferred between the meristem layers via the direct symplasmic route, without passage through the cell membrane (Jackson et al. 1994; Mezitt and Lucas 1996), or diffuse through the apoplasmic space (Becraft 1995; Furner et al. 1996). The polar interaction in case of globosa is thought to result from the trafficking of the GLOBOSA (GLO) itself, moving via secondary plasmodesmata towards the tunica (Perbal et al. 1996). Within the tunica lateral spread of GLO was severely restricted. This was attributed to the assumptive absence of secondary plasmodesmata. However, a symplasmetric map, visualizing the distribution of plasmodesmata types in the meristem, shows that many secondary plasmodesmata are present within the tunica (Fig. 6; Bergmans et al. 1997), indicating that in principle all cells can be reached via secondary plasmodesmata. Therefore, only an set of parallel cell lineages in part of a bisemetrical meristem may have restricted movement of GLO in one direction. Macromolecular transfer through plasmodesmata in the AM thus seems feasible. It remains to be dem-

Fig. 6A-D. Construction of a symplasmetric map of the duplex shoot apical meristem $(A M)$ of angiosperms. A Longitudinal section through the apex. $C$ Corpus; $T$ two-layered tunica $\left(L_{1}, L_{2}\right)$. B Surface projection of the AM with initial cell $(I)$, derived sector $(S)$, and two longitudinal sections through the sector (arrows 1 and 2). $\mathrm{C}$ Reconstruction of layered sectors; $L_{1}, L_{2}$ tunica layers; $L_{3}$ corpus; $I$ initial cell; $S$ derived sector. D Symplasmetric map representing a sector in $\mathrm{L}_{2}$ (in $\mathrm{L}_{1}$ outer-wall plasmodesmata are absent). As an example, two cross-sections (arrows 1, 2 in B) through layer $\mathrm{L}_{2}$ are depicted with anticlinal and periclinal cell interfaces which possess exclusively primary (small arrow) or secondary (small dotted arrow) plasmodesmata. Note that the plasmodesmata are exclusively secondary at $\mathrm{L}_{2} / \mathrm{L}_{1}$ and between $\mathrm{L}_{2}$ and corpus. (Bergmans et al. 1997)
A

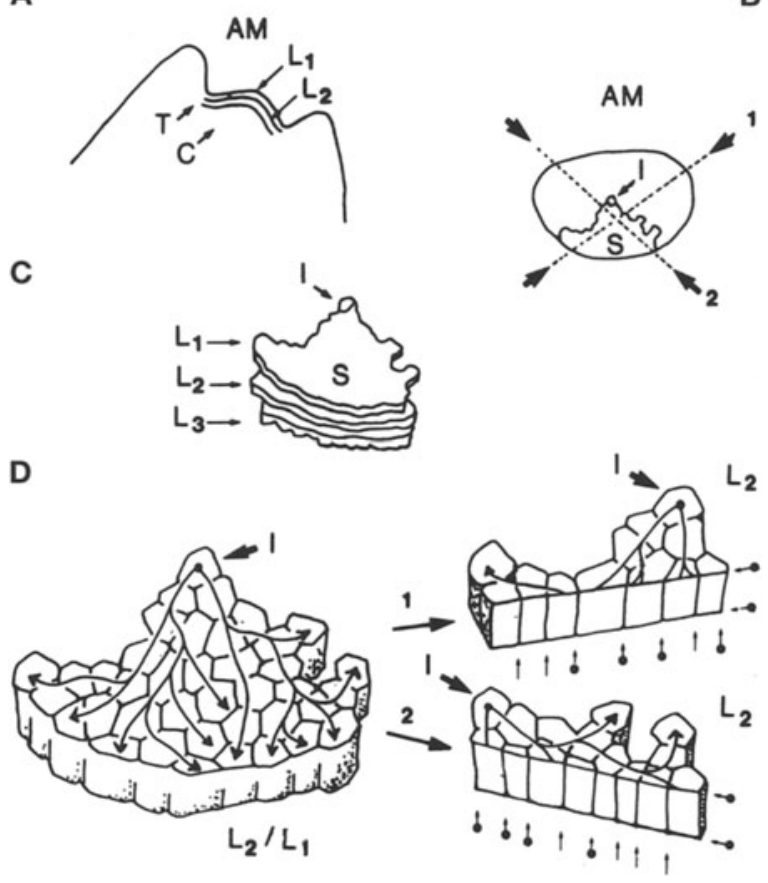


onstrated, however, whether GLO, DEF and FLo themselves move from cell-to-cell instead of mediating factors and, if so, through which pathways.

Understanding how the complex interactions involved in morphogenesis lead to an integrated output requires a concept of the AM as a whole. Such concepts are available from, for example, traditional embryology which explained morphogenesis in terms of dynamic systems and morphogenetic fields.

\subsection{A Morphogenetic Field}

The AM resembles the morphogenetic field of animal systems (Holder 1979; Rinne and van der Schoot 1998), which is defined as "an area whose cells can cooperatively bring about a distinct set of structures" (Müller 1997). The AM, like the morphogenetic fields in animals (de Robertis et al. 1991; Müller 1997), has remarkable regenerative and regulative capacities (e.g. Steeves and Sussex 1989; Sussex 1989). For animal systems these properties have been explained in a variety of ways (see e.g. Müller 1997). According to the positional information model (PI model), gradients of morphogens specify cellular differentiation (e.g. Wolpert 1969, 1989). Gradients may be composed of immobile substances, fixed either inside the cells or at the extracellular matrix. Alternatively, gradients may be formed by morphogens which diffuse from a source to a sink region. The cells in the source-to-sink paths may consume morphogen, and thereby decrease the size of the morphogenetic field. The presence of such gradient fields prepatterns the morphogenetic field (Crick 1970; Müller 1997) and permits cells to establish their position with regard to the boundaries of the field. Dependent on their developmental history cells interpret gradients and record them as positional values. Collectively, these values could function as a tissue memory, used for regeneration and further development (Holder 1979; Wolpert 1969, 1989).

In animals and plants the diffusion paths of morphogens are assumed to be situated in the extracellular space, but gap junctions (Wolpert 1978) and plasmodesmata (Pitts 1990) suit as well the formation of morphogen gradients in a symplasmic space. In the proliferating AM cells are displaced towards the periphery and may then pass through sequential states. If each cell broadcasts signals in correspondence to its state, a process of active symplasmic conversation would continuously update the information on cellular positions in a cooperatively generated field. The behaviour of an individual cell would then depend on that of all other cells, a condition which goes beyond the PI model, however, the AM does not show a gradual change in its cellular characteristics, but instead a rather discrete zonation pattern. Like indicated earlier (Sect. 3.1), the transient closure of plasmodesmata at specific demarcation lines within the AM may keep physiologically distinct areas apart.

\subsection{Symplasmic Fields}

Evidence is now emerging that the coordination of morphogenesis requires the dynamic subdivision of the symplasmic space of the AM into diffusion compartments by the position-dependent closure of plasmodesmata, (van der Schoot et al. 1991; van der Schoot and Rinne 1997; Rinne and van der Schoot 1998). Symplasmic compartments 
were first found in mature tissues of Elodea and were referred to as "symplastic domains" (Erwee and Goodwin 1985). They serve the physiological stabilization of tissues by the internal redistribution of metabolites (van der Schoot and van Bel 1990; Lucas et al. 1993). Since the AM is different from mature tissues in that it is continuously renewing its cellular composition, these "domains" may be better envisaged as "fields" (van der Schoot and Rinne 1997; Rinne and van der Schoot 1998). This would also be in line with the embryological concepts (see e.g. Holder 1979). The symplasmic fields can be visualized in the actively proliferating AM by iontophoretic microinjection of Lucifer Yellow CH ( $\mathrm{LYCH}$ ), a fluorescent membrane-impermeable probe (Rinne and van der Schoot 1998). When injected into a single cell, its movement to neighbouring cells reveals the symplasmic pathways open for the diffusion of small metabolites, hormones and other regulators. In the tunica of birch and potato, the distinct symplasmic fields corresponded in position to the peripheral part and the central part of the AM (Fig. 7). The symplasmic fields appeared to be dynamic, and they showed spatiotemporal alterations which corresponded to morphogenetic events at the AM (van der Schoot and Rinne 1996, 1997; Rinne and van der Schoot 1998). These fields harbour gradients of potential morphogens small enough to move through plasmodesmata by diffusion (up to $1 \mathrm{kDa}$ ). The membrane potentials $\left(\mathrm{E}_{\mathrm{m}}\right)$ recorded from the individual

A

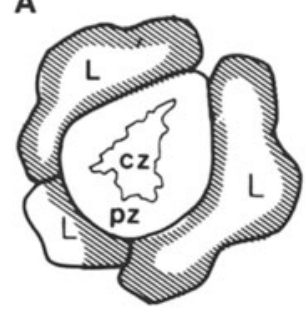

C

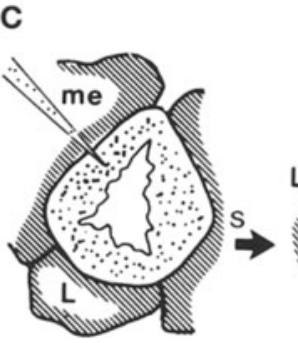

B

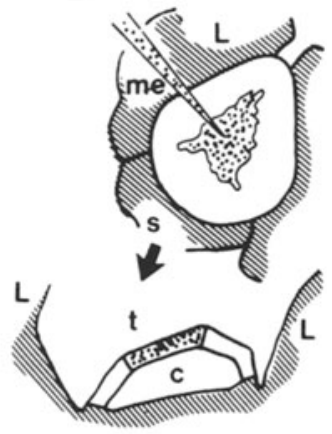

Fig. 7A-C. A schematic illustration of symplasmic fields in the tunica of the shoot apical meristem (AM) in birch (Betula pubescens Ehrh.), and their correspondence to classical cytohistological zones. A AM in surface view showing central zone $(c z)$ and peripheral zone $(p z)$. The precise outline of the border was revealed by microinjection experiments shown in B and C. Symplasmic fields were visualized by the cell-to-cell movement of LYCH (dotted areas), iontophoretically injected into a single tunica cell in the centre (B) or periphery (C) of the AM. The symplasmic field at the pz corresponded to the organogenetic ring, and the symplasmic field at the cz corresponded to the uncommitted cells at the AM centre; $s$ longitudinal section through the injected AMs showing the presence of LYCH in the tunica $(t)$ cells; $c$ corpus; $l$ leaf primordia; me microelectrode. (After Rinne and van der Schoot 1998) 
AM cells were field-specific, demonstrating that these fields also function as metabolic working units. The plasmodesmata between the symplasmic fields and towards the primordia and the corpus were closed for LYCH. The size of the central symplasmic field was rather constant, even though the constituent cells proliferate. This means that plasmodesmata closure between the central and peripheral field is transient and position-dependent, and under the control of a yet hypothetical mechanism anchored in the initial cells. The initial cells may therefore organize the central fields of the tunica and the corpus, which collectively may represent a shoot organizer (van der Schoot and Rinne 1996; Rinne and van der Schoot 1998).

Some mutants may provide access to the mechanism which are involved in formation of symplasmic fields. The petunia mutant no apical meristem (nam) is defective in a gene, the product of which demarcates the boundaries between the primordia and the AM proper (Souer et al. 1996), and NAM may hence be involved in either the establishment of boundaries between symplasmic fields or in mediating field-field interactions. Similarly, the Arabidopsis mutants Fey and Dip which early in development loose the ability to maintain a central undifferentiated AM area (Medford et al. 1992), might be impaired in the mechanism which closes the plasmodesmata at the field boundaries.

The symplasmic fields harbour signal networks and may therefore be depicted as circuits. Signal coupling of these separate circuits can be achieved by the exchange of macromolecular signals after transient gating of the closed plasmodesmata, or by the exchange of lipophilic signalling molecules via the ER (Grabski et al. 1993). Cells, which are forced out of their position, will join a new subcircuit and their differentiation becomes redirected. Alternatively, cells of the central tunica field, for example, may be released towards the periphery, and thereby carry specific information into an expanding part of the peripheral field. Upon symplasmic integration into this area, they may start to organize their surroundings in order to create a primordium (van der Schoot and Rinne 1996; Rinne and van der Schoot 1998). Resumingly, the AM may harbour distinct but overlapping signal circuits, which serve the execution of the conflicting tasks of patterning and self-maintenance, and which may also function in phyllotactic patterning.

\subsection{Symplasmic Field Transitions}

Since the AM responds to environmental signals with dramatic developmental changes, e.g. dormancy development and flower formation, transitions in the symplasmic network of the AM are likely to occur as well. For dormancy this has been confirmed by the demonstration that the patterns of the symplasmic fields in the AM change during development of the dormant state in birch (Rinne and van der Schoot 1998) and potato (C. van der Schoot, unpub.). In birch, the symplasmic fields disappeared prior to visible cessation of growth in response to short photoperiod, suggesting a link between phytochrome action and symplasmic uncoupling of AM cells. This was due to physical blockage of all plasmodesmata in the entire AM by the formation of callosecontaining sphincters (Fig. 5), thereby preventing any form of symplasmic communication. How phytochrome action relates to mechanisms that control plasmodesmata remains to be established. In the dormant potato AM, the majority of the plasmodes- 
mata was closed by sphincters, but part was widened due to the removal of the ER (van der Schoot 1996). The latter permitted the cell-to-cell movement of LYCH-dextran molecules whose size prevents its movement through normal plasmodesmata. Such an increase in the size exclusion limit of plasmodesmata possibly creates a partly syncytial state, which renders communication ineffective due to loss of selectivity. These two examples of symplasmic non-communication during dormancy development demonstrate the sensitivity of the symplasm to environmental factors, and they support the assumption that symplasmic communication is indispensable in morphogenesis.

There is no experimental evidence so far that the symplasm alters its overall organization during the transition to flowering. Transformation of an AM into an inflorescence meristem does not alter the zonation pattern (e.g. Bernier 1962). This is expected, since an undifferentiated centre is retained as long as the inflorescence develops. Therefore, the inflorescence meristem may also possess a central and a peripheral field. When a terminal flower is formed, zonation disappears and the meristem centre is used up (Takimoto 1969). The disappearance of the zonation pattern during the transition may indicate that the symplasmic fields are reorganized and gradually used up. We suggest that a stable central symplasmic field is characteristic of an open growth process; when the AM (or inflorescence meristem) commits itself to end differentiation, becoming a flower or a thorn, the central symplasmic fields of the tunica and corpus give up their sovereignty.

\section{$4 \quad$ Concluding Remarks}

The various types of shoot AM - monoplex, simplex and duplex - and the specific ways in which they organize the symplasm by cell divisions, are dependent on the capacity to unite non-daughter cells by secondary plasmodesmata. Phylogenetically, the capacity to form plasmodesmata through existing walls permitted the duplex AM construction, found in all angiosperms. The emerging picture of the duplex AM is that of a dynamically organized unit, which orchestrates primary morphogenesis and simultaneously maintains itself. The implementation of these conflicting tasks is achieved by subdividing the AM by means of plasmodesmata closure into physiologically distinct symplasmic compartments, which might interact via transiently gating plasmodesmata. The challenge for the future will be to elucidate how the AM, as a symplasmically integrated whole, maintains local differences in cell physiology and gene expression, and how it alters them in response to signals from the rest of the plant or the environment during development.

Acknowledgements. We thank R.W. Goldbach (Agricultural University Wageningen) and A.D. de Boer (ATO-DLO, Wageningen) for support. The Academy of Finland is acknowledged for an 'International Cooperation Grant' to P. R.

\section{References}

Becraft PW (1995) Intercellular induction of homeotic gene expression in flower development. Trends Genet $11: 253-255$

Berger F, Taylor A, Brownlee C (1994) Cell fate determination by the cell wall in early Fucus development. Science 263:1421-1423 
Bergmans A, de Boer D, van Bel A, van der Schoot C (1993) The initiation and development of Iris flowers: permeability changes in the apex symplasm. Flowering Newsl 16:19-26

Bergmans ACJ, de Boer AD, Derksen JWM, van der Schoot C (1997) The symplasmic coupling of $\mathrm{L}_{2-}$ cells diminishes in early floral development of Iris. Planta 203:245-252

Bernier G (1962) Evolution of the apical meristem of Sinapis alba L. (long-day plant) in long days, in short days and during the transfer from short days to long days. Caryologia 15:303-325

Carpenter R, Coen ES (1995) Transposon induced chimeras show that floricaula, a meristem identity gene, acts non-autonomously between cell layers. Development 121:19-26

Carr DJ (1976) Plasmodesmata in growth and development. In: Gunning BES, Robards AW (eds) Intercellular communication in plants: studies on plasmodesmata. Springer, Berlin Heidelberg New York, pp 243-289

Coen ES, Meyerowitz EM (1991) The war of the whorls: genetic interactions controlling flower development. Nature 353:31-36

Cooke TJ, Tilney MS, Tilney LG (1996) Plasmodesmatal networks in apical meristems and mature structures: geometric evidence for both primary and secondary formation of plasmodesmata. In: Smallwood M, Knox JP, Bowles DJ (eds) Membranes: specialized functions in plants. Bios Scientific Publishers, Oxford, pp 471-488

Crick FHC (1970) Diffusion in embryogenesis. Nature 225:546-548

de Robertis EM, Morita EA, Cho KWY (1991) Gradient fields and homeobox genes. Development 112:669-678

Ding B, Haudenshield JS, Hull R, Wolf S, Beachy RN, Lucas WJ (1992) Secondary plasmodesmata are specific sites of localization of the tobacco mosaic virus movement protein in transgenic tobacco plants. Plant Cell 4:915-928

Ding B, Haudenshield JS, Willmitzer L, Lucas WJ (1993) Correlation between arrested secondary plasmodesmal development and onset of accelerated leaf senescence in yeast acid invertase transgenic tobacco plants. Plant J 4:179-189

Ehlers K, Kollmann R (1996) Formation of branched plasmodesmata in regenerating Solanum nigrum-protoplasts. Planta 199: 126-138

Erwee MG, Goodwin PB (1985) Symplast domains in extrastellar tissues of Egeria densa Planch. Planta 163:9-19

Fleming AJ, Mandel T, Hofmann S, Sterk P, de Vries SC, Kuhlemeier C (1992) Expression patterns of a tobacco lipid transfer protein gene within the shoot apex. Plant J 2:855-862

Fleming AJ, Mandel T, Roth I, Kuhlemeier C (1993) The patterns of gene expression in the tomato shoot apical meristem. Plant Cell 5:297-309

Franceschi VR, Ding B, Lucas WJ (1994) Mechanism of plasmodesmata formation in characean algae in relation to evolution of intercellular communication in higher plants. Planta 192:347-358

Furner IJ, Ainscough JF-X, Pumfrey JA, Petty LM (1996) Clonal analysis of the late flowering fca mutant of Arabidopsis thaliana: cell fate and cell autonomy. Development 122:1041-1050

Gifford EM, Corson GE (1971) The shoot apex of seed plants. Bot Rev 37:143-229

Grabski S, de Feijter AW, Schindler M (1993) Endoplasmic reticulum forms a dynamic continuum for lipid diffusion between contiguous soybean root cells. Plant Cell 5:23-35

Green PB (1992) Pattern formation in shoots: a likely role for minimum energy configurations of the tunica. Int J Plant Sci Suppl 153:59-75

Green PB, Steele CS, Rennich SC (1996) Phyllotactic patterns: a biophysical mechanism for their origin. Ann Bot 77:515-527

Gunning BES, Robards AW (1976) Plasmodesmata: current knowledge and outstanding problems. In: Gunning BES, Robards AW (eds) Intercellular communication in plants: studies on plasmodesmata. Springer, Berlin Heidelberg New York, pp 297-311

Hake S, Freeling M (1986) Analysis of genetic mosaics shows that the extra epidermal cell divisions in Knotted mutant maize plants are induced by adjacent mesophyll cells. Nature 320:621-623

Hantke SS, Carpenter R, Coen ES (1995) Expression of floricaula in single cell layers of periclinal chimeras activates downstream homeotic genes in all layers of floral meristems. Development 121: 27-35

Holder N (1979) Positional information and pattern formation in plant morphogenesis and a mechanism for the involvement of plant hormones. J Theor Biol 77:195-212

Jackson D, Veit B, Hake S (1994) Expression of maize KNOTTED 1 related homeobox genes in the shoot apical meristem predicts patterns of morphogenesis in the vegetative shoot. Development 120: 405-413

Jones MGK (1976) The origin and development of plasmodesmata. In: Gunning BES, Robards AW (eds) Intercellular communication in plants: studies on plasmodesmata. Springer, Berlin Heidelberg New York, pp 81-105

Kaplan DR, Hagemann W (1991) The relationship of cell and organism in vascular plants. Are cells the building blocks of plant form? BioScience 41:693-703 
Kelly AJ, Zagotta MT, White RA, Chang C, Meeks-Wagner DR (1990) Identification of genes expressed in the tobacco shoot apex during the floral transition. Plant Cell 2:963-972

Kollmann R, Glockmann C (1985) Studies on graft unions. I. Plasmodesmata between cells of plants belonging to different unrelated taxa. Protoplasma 124:224-235

Lucas WJ, Ding B, van der Schoot C (1993) Plasmodesmata and the supracellular nature of plants. New Phytol 125:435-476

Lucas WJ, Bouché-Pillon S, Jackson DP, Nguyen L, Baker L, Ding B, Hake S (1995) Selective trafficking of KNOTTED 1 homeodomain protein and its mRNA through plasmodesmata. Science 270: 1980-1983

Medford JI, Behringer FJ, Callos JD, Feldmann KA (1992) Normal and abnormal development in the Arabidopsis vegetative shoot apex. Plant Cell 4:631-643

Menzel G, Apel K, Melzer S (1996) Identification of two MADs box genes that are expressed in the apical meristem of the long-day plant Sinapis alba in transition to flowering. Plant J 9:399-408

Mezitt LA, Lucas WJ (1996) Plasmodesmal cell-to-cell transport of proteins and nucleic acids. Plant Mol Biol 32:215-273

Müller WA (1997) Developmental biology. Springer, Berlin Heidelberg New York

Newman IV (1965) Pattern in the meristems of vascular plants. III. Pursuing the patterns in the apical meristem where no cell is a permanent cell. Linn Soc (Bot) 59:185-214

Olesen P, Robards AW (1990) The neck region of plasmodesmata: general architecture and functional aspects. In: Robards AW, Lucas WJ, Pitts JD, Jongsma HJ, Sprey DC (eds) Parallels in cell-to-cell junctions in plants and animals. Springer, Berlin Heidelberg New York, pp 145-170

Perbal M-C, Haughn G, Seadler H, Schwarz-Sommer Z (1996) Non-cell-autonomous function of the Antirrhinum floral homeotic proteins DEFICIENS and GLOBOSA is exerted by their polar cell-to-cell trafficking. Development 122:3433-3441

Pitts JD (1990) Junctional communication: the role of communication compartments in complex multicellular organisms. In: Robards AW, Lucas WJ, Pitts JD, Jongsma HJ, Sprey DC (eds) Parallels in cell-to-cell junctions in plants and animals. Springer, Berlin Heidelberg New York, pp 53-62

Pri-Hadash A, Hareven D, Lifschitz E (1992) A meristem-related gene from tomato encodes a dUTPase: analysis of expression in vegetative and floral meristems. Plant Cell 4:149-159

Raghavan V (1989) Developmental biology of fern gametophytes. Cambridge University Press, Cambridge

Rinne P, van der Schoot C (1998) Symplasmic fields in the tunica of the shoot apical meristem coordinate morphogenetic events. Development 125:1477-1485

Robards AW (1976) Plasmodesmata in higher plants. In: Gunning BES, Robards AW (eds) Intercellular communication in plants: studies on plasmodesmata. Springer, Berlin Heidelberg New York, pp $15-53$

Roberts K (1994) The plant extracellular matrix: in a new expansive mood. Curr Opin Cell Biol 6: 688-694

Sachs T (1991) Pattern formation in plant tissues. Cambridge University Press, Cambridge

Sawhney VK, Rennie PJ, Steeves TA (1981) The ultrastructure of the central zone cells of the shoot apex of Helianthus annuus. Can J Bot 59:2009-2015

Seagull RW (1983) Differences in the frequency and disposition of plasmodesmata resulting from root-cell elongation. Planta 159:497-504

Selker JML, Steucek GL, Green PB (1992) Biophysical mechanisms for morphogenetic progressions at the shoot apex. Dev Biol 153:29-43

Souer E, van Houwelingen A, Kloos D, Mol J, Koes R (1996) The no apical meristem gene of petunia is required for pattern formation in embryos and flowers and is expressed at meristem and primordia boundaries. Cell 85:159-170

Steeves TA, Sussex IM (1989) Patterns in plant development. Cambridge University Press, Cambridge

Sussex IM (1952) Regeneration of the potato shoot apex. Nature 170:755-757

Sussex IM (1989) Developmental programming of the shoot meristem. Cell 56:225-229

Takimoto A (1969) Pharbitis nil Chois. In: Evans LT (ed) The induction of flowering. Some case histories. Macmillan, Melbourne, pp 90-115

Tilney LG, Cooke TJ, Connelly PS, Tilney MS (1990) The distribution of plasmodesmata and its relationship to morphogenesis in fern gametophytes. Development 110:1209-1221

Tilney-Bassett RAE (1986) Plant chimeras. Edward Arnold, London

Turner A, Wells B, Roberts K (1994) Plasmodesmata of maize root tips: structure and composition. J Cell Sci 107:3351-3361

van der Schoot C (1994) The role of symplasmic organization and cell-cell communication in dormancy. In: Lang GA (ed) 1st Int Symp on Plant Dormancy, 7-10 August 1994, Oregon State University, Corvallis, Oregon, p 30

van der Schoot C (1996) Dormancy and symplasmic networking at the shoot apical meristem. In: Lang GA (ed) Plant dormancy. CAB Int, Wallingford, pp 59-81 
van der Schoot C, Rinne P (1996) Symplasmic networking and autopoiesis at the shoot apical meristem. In: Proc 10ème Réunion Groupe d'Etude de l'Arbre, 16-17 April 1996, Angers, pp 36-55

van der Schoot C, Rinne P (1997) Symplasmic networking at the shoot apical meristem. In: van Montagu M, Micol JL (eds) Worksh on plant morphogenesis, Madrid 20-22 Oct 1997. Instituto Juan March de Estudios e Investigaciones. Centro de Reuniones Internacionales Sobre Biología, no 72, pp 51-52

van der Schoot C, van Bel AJE (1990) Mapping membrane potential differences and dye-coupling in internodal tissues of tomato (Solanum lycopersicum L). Planta 182:9-21

van der Schoot C, Drent P, Thiel F, Clerkx A, Boekestein B (1991) Functional structure of the symplast in resting apices of Solanum tuberosum L. Plant Physiol Suppl 96:154

van der Schoot C, Dietrich MA, Storms M, Verbeke JA, Lucas WJ (1995) Establishment of a cell-to-cell communication pathway between separate carpels during gynoecium development. Planta 195: 450-455

Weigel D, Clark SE (1996) Sizing up the floral meristem. Plant Physiol 112:5-10

Wolpert L (1969) Positional information and the spatial pattern of cellular differentiation. J Theor Biol 25: $1-47$

Wolpert L (1978) Gap junctions: channels for communication in development In: Feldman J, Gilula NB, Pitts JD (eds) Intercellular junctions and synapses. Receptors and recognition, series B, vol 2. Chapman and Hall, London, pp 83-96

Wolpert L (1989) Positional information revised. Development Suppl 3-12 